$\Rightarrow$ PROSTATE CANCER

\title{
Postoperative RT response prediction
}

A newly developed 24-gene score can predict which patients benefit from radiotherapy (RT) after radical prostatectomy based on tumour gene expression profiles.

RT can be a valuable option after surgical treatment for prostate cancer but selecting those patients who would benefit most from this adjuvant therapy is difficult. In a new retrospective analysis, researchers used data from five previously published studies to create a predictive tool. All men had undergone radical prostatectomy and were matched according to whether they had subsequent RT and to clinicopathological variables, such as Gleason score, margin status and lymph node invasion. Incidence of distant metastasis on imaging was the primary end point of their analysis.

The training cohort (196 men) was used to generate the Post-Operative Radiation Therapy Outcomes Score (PORTOS), an expression signature of 24 genes involved in DNA damage, radiation and immune response. Of the 39 men who had a high PORTOS, those who had or had not received RT had a 10-year metastasis rate of $5 \%$ or $63 \%$, respectively (HR 0.12, 95\% Cl 0.03-0.41; $P<0.0001$ ). By contrast, among 157 men with a low PORTOS, $57 \%$ of those who had but only $31 \%$ of those who had not received RT had distant metastasis at 10 years (HR 2.5, 95\% Cl 1.6-4.1; $P<0.0001)$. Overfitting might have contributed to this counterintuitive result, according to the study authors.

In the validation cohort of 330 men, the results for men with a high PORTOS were confirmed ( $4 \%$ versus $35 \%$ with metastasis at 10 years; HR 0.15, 95\% Cl 0.04-0.60; $\mathrm{P}=0.0020$ ). In the low PORTOS group, incidence of 10 -year metastasis was $32 \%$ regardless of RT.

The researchers also tested whether three scores that are prognostic for radical prostatectomy outcomes (Decipher, $\mathrm{mCCP}$ and (APRA-S) could predict postoperative RT responses in the validation cohort. Using different cut-off values, the team found no statistically significant interaction between the three scores and RT.

Clemens Thoma

ORIGINAL ARTICLE Zhao, S. G. et al. Development and validation of a 24-gene predictor of response to postoperative radiotherapy in prostate cancer: a matched, retrospective analysis. Lancet Oncol. http://dx.doi.org/10.1016/S14702045(16)30491-0 (2016) 